\title{
Percutaneous Embolization of Scalp Arteriovenous Malformation Using New Liquid Embolic Agent, SQUID: A Technical Report
}

\author{
Santhosh Kumar Kannath ${ }^{1}$ Jayadevan Enakshy Rajan \\ ${ }^{1}$ Department of Imaging Sciences and Interventional Radiology, \\ Neurointervention Center, Sree Chitra Tirunal Institute of Medical \\ Sciences and Technology, Trivandrum, Kerala, India \\ J Clin Interv Radiol ISVIR 2017;1:171-174.
}

\author{
Address for correspondence Jayadevan Enakshy Rajan, DM, \\ Department of Imaging Sciences and Interventional Radiology, \\ Neurointervention Center, Sree Chitra Tirunal Institute of Medical \\ Sciences and Technology, Trivandrum 695011, Kerala, India \\ (e-mail: drsanthoshkannath@gmail.com).
}

\author{
Abstract \\ Keywords \\ - scalp arteriovenous \\ malformation \\ - ethylene vinyl alcohol \\ copolymer \\ - SQUID \\ - percutaneous \\ embolization
}

Scalp arteriovenous malformations (AVMs) are rare extracranial vascular malformations that are traditionally treated by surgical excision or by combined endovascularsurgical approaches. We describe percutaneous treatment of scalp AVM, using a new liquid embolic agent, SQUID. Because of its nonadhesive property, prolonged injection and complete obliteration of scalp AVM is feasible. The cast formed is aesthetically appealing to the patient, and it may obviate the need for further surgical excision. It could be considered as the primary treatment option for patients unwilling for surgery.

\section{Introduction}

Scalp arteriovenous malformations (AVMs) are rare extracranial vascular malformations. In the past, etiologies such as trauma, idiopathic, and iatrogenic have been considered. ${ }^{1}$ Traditionally, these lesions are treated by surgical excision or by combined endovascular-surgical approaches. ${ }^{2-5}$ Recent reports describe the treatment of scalp AVM with nonadhesive liquid embolic agent such as Onyx (Covidien/Medtronics, Irvine, California, United States). ${ }^{6-9}$ The existing literature pertains to transarterial as well as percutaneous embolization of scalp AVM. The data on percutaneous approaches are limited, and there are no reports as yet on the use of the new liquid embolic agent, SQUID (Emboflu, Switzerland) in the treatment of scalp AVM. We describe the technical details and advantages of percutaneous treatment of scalp AVM with SQUID and briefly review the existing literature on application of ethylene vinyl alcohol $(\mathrm{EVOH})$ co-polymer in the treatment of this extracranial vascular malformation.

received

January 16, 2017

accepted after revision

May 5, 2017

published online

September 1, 2017

\section{Case Description}

A middle-aged man presented with complaints of bruit over the left mastoid region, which increased in severity over the last 6 months. Clinical examination revealed pulsatile soft swelling and thrill over the left mastoid region, and it was confirmed to be scalp AVM by Doppler evaluation. He was considered for definitive therapy as the bruit was troublesome. Under general anesthesia, he underwent angiographic evaluation, which revealed predominantly fistulous AVM fed by hypertrophied feeders of superficial temporal and occipital arteries and draining into ectatic superficial parietooccipital vein. The AVM was confined to the scalp region with no communication to intracranial venous structures. Following angiography, the dilated venous sac of the AVM was accessed percutaneously using a $16 \mathrm{G}$ cannula under roadmap guidance from external carotid artery injection. The cannula was advanced further into the vein using a 0.035 -in hydrophilic guide wire and secured with a sterile tape. A rotating hemostatic valve was attached to the hub of cannula

Copyright ( 2017 by Indian Society of Vascular and Interventional Radiology 


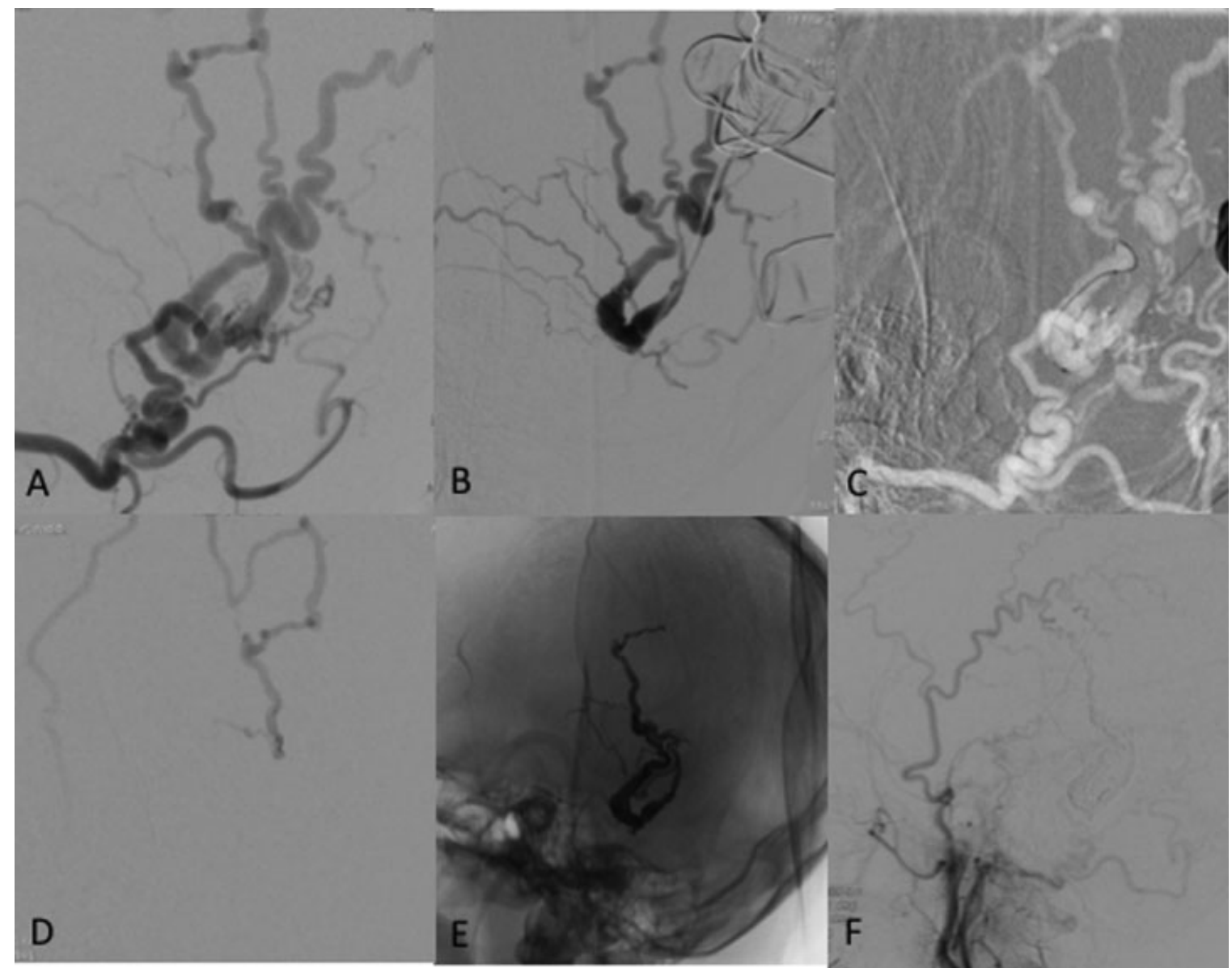

Fig. 1 Selective occipital angiogram (A) shows a scalp AVM in the mastoid region. The venous side of the AVM is accessed percutaneously by $16 \mathrm{C}$ (B) and a microcatheter is navigated close to the nidus under roadmap guidance (C). Following initial microcatheter angiogram (D), the AVM is embolized with SQUID embolic agent. The embolic cast $(E)$ and obliterated AVM $(F)$ is demonstrated.

to avoid back bleed. The vein was later catheterized with an Echelon (Covidien/Medtronics) microcatheter over a Traxcess (MicroVention, Tustin, California, United States) microguidewire, and the tip of the microcatheter was placed closed to the nidus. The microcatheter dead space was flushed with DMSO, and the vein was slowly occluded with $3.5 \mathrm{~mL}$ of SQUID 18 embolic agent over 20 minutes. Because of high flow, temporary flow arrest was obtained to form an initial cast, and subsequent embolization allowed permanent stabilization of the embolic agent. Retrograde embolization into the nidus and arterial feeders were also noted during the embolization. Check angiogram revealed complete obliteration of the fistula, and the microcatheter and cannula were removed without any complications (-Fig. 1). Postprocedure, small boggy swelling was noted at the site of the AVM without any tenderness or skin discolorations. He was discharged on postprocedure day 2, and clinical and Doppler evaluation revealed no residual AVM or nodular embolic cast at 1-year follow-up.

\section{Discussion}

The clinical presentation of scalp AVM may vary from benign features such as pulsatile swelling and tinnitus to malignant symptoms such as life-threatening hemorrhage and scalp necrosis. ${ }^{1}$ The indications for treatment include recurrent or massive hemorrhage from the AVM, bothersome symptoms, or cosmetic concerns. Traditionally, the scalp AVMs have been treated by surgery that included radical surgical excision and feeding artery ligation. The surgery is often complicated by major intraoperative bleeding and the surgeon attempts to achieve hemostasis by feeding artery ligation, tourniquets, or clamps. Incomplete excision results in residual or recurrent AVMs and further complicates future surgical or endovascular management. ${ }^{2}$ Also, scalp reconstruction is an important consideration when the surgical defect is large. ${ }^{3}$

Over the last two decades, endovascular management has been increasingly reported as the mainstay therapy or surgical adjunct in the treatment of scalp AVMs. The endovascular routes are varied and the choices of embolic agents are myriad. Transarterial, transvenous, and percutaneous approaches with different embolic agents such as polyvinyl alcohol (PVA) particles, n-butyl cyanoacrylates (n-BCA), and EVOH copolymers are all described in the literature. $^{1,3-9}$ The endovascular treatment is particularly attractive as it is minimally invasive and could be repeated several times to achieve adequate devascularization prior to 
definitive surgical excision. Direct puncture and n-butyl cyanoacrylate embolization of scalp AVMs have been found to be safe and effective in achieving devascularization prior to surgery. Although complete obliteration is achievable in certain situations, surgical resection is often performed to avoid recurrence as well as cosmetic disfigurement due to lumpy glue cast. ${ }^{4,5}$

Recently, the EVOH co-polymer-based liquid embolic agent, Onyx has been shown to be effective in the treatment of scalp AVMs. ${ }^{6-8}$ EVOH copolymer is nonadhesive, and hence it permits prolonged injection time without the fear of catheter entrapment. It precipitates along the vessel wall and slowly permeates into various nidal and venous compartments, and thus it can achieve higher obliteration rates compared with n-BCA. The progress of embolization could be assessed with repeated angiograms and the operator could preferentially fill the residual nidus or avoid important vascular segment by "wait and push" technique. Flow reduction is necessary to form an initial stable plug, and this is achieved by several ways such as proximal arterial balloon occlusion, retrograde venous balloon occlusion, coiling of selective venous tributaries followed by Onyx embolization, and coiling of venous sac or with tourniquets. ${ }^{6-11}$ Postembolization, boggy swelling is evident over the embolized AVM, which is aesthetically acceptable to patients, especially if they wish to avoid further surgery. ${ }^{12}$ SQUID is a recently introduced liquid embolic agent that has chemical composition similar to Onyx. The notable difference of SQUID from Onyx is in the particulate size of the tantalum, which provides radio-opacity to the embolic material. The micronization of tantalum in SQUID retards its precipitation with in the microcatheter, and thus it reduces the risk of microcatheter blockage, especially during prolonged injection periods. The embolic agent has received CE approval for the treatment of cerebral AVM, and it is currently available in Europe and some Asian countries.

The success of endovascular treatment depends on three factors: (1) the microcatheter tip as close to fistula as possible to enable optimal penetration of embolic agent into the nidus and the venous sac; (2) adequate flow arrest to allow stable embolic plug formation and prevent inadvertent pulmonary embolization; and (3) appropriate choice of embolic agent to allow prolonged injection and avoid catheter entrapment. Transarterial approach can be cumbersome as the arteries are often extremely tortuous and serpiginous and precludes favorable microcatheter position close the nidus. Similarly, venous approach too can be difficult due to the presence of sacs, ectasias, or ramifications. Moreover, these approaches need flow arrest using balloons or tourniquets to allow stable cast formation and prevent inadvertent migration. ${ }^{6,8}$

There is only sparse literature on percutaneous direct puncture Onyx embolization of scalp AVMs, and there are no reports as yet on usefulness of SQUID in the treatment of scalp AVMs. SQUID is available in two compositions differing in viscosity (SQUID 12 and SQUID 18). The tantalum powder in SQUID is micronized to avoid precipitation and catheter clogging during prolonged injections. We percutaneously accessed the principal draining vein distal to the nidus and cannulated the foot of nidus using a microcatheter. A 16G cannula allows the introduction of microcatheter and this cannula could be safely taped onto the scalp. Flow arrest could be achieved by taping the cannula tightly, and secondary veins could be compressed manually or with temporary tourniquets during the procedure. Once stable embolic cast is formed, flow limitation is not mandatory. Additionally, it allows prolonged injection of embolic agent without serious concern of the degree of reflux because, if the catheter is entrapped, it could be cut at the puncture site and left in situ. The limitation of this technique includes operator radiation exposure during the vascular access; however, it is much less compared with direct access and glue embolization as the longer catheter length allows the operator to stay away from the X-ray tube during embolization. Risk of blackish staining over the skin exists, though we have not observed it in our patient.

\section{Conclusion}

Percutaneous direct puncture embolization using SQUID is an additional treatment option for patients with scalp AVMs. It avoids several limitations associated with other therapeutic options and could be considered as the primary treatment option for patients unwilling for surgery. Long-term follow-up and larger experience are needed to confirm these findings.

\section{Contributions of Authors}

Santhosh Kumar Kannath contributed in concept, design, data analysis, data interpretation, manuscript preparation, and critical revision, and Jayadevan Enakshy Rajan contributed in data analysis, data interpretation, manuscript preparation, and critical revision.

\section{Conflicts of Interest}

The authors have no personal or financial conflict of interest to disclose.

\section{References}

1 Gupta AK, Purkayastha S, Bodhey NK, et al. Endovascular treatment of scalp cirsoid aneurysms. Neurol India 2008;56(02): $167-172$

2 Fisher-Jeffes ND, Domingo Z, Madden M, de Villiers JC. Arteriovenous malformations of the scalp. Neurosurgery 1995;36(04): 656-660, discussion 660

3 Nagasaka S, Fukushima T, Goto K, Ohjimi H, Iwabuchi S, Maehara F. Treatment of scalp arteriovenous malformation. Neurosurgery 1996;38(04):671-677, discussion 677

4 Han MH, Seong SO, Kim HD, Chang KH, Yeon KM, Han MC. Craniofacial arteriovenous malformation: preoperative embolization with direct puncture and injection of n-butyl cyanoacrylate. Radiology 1999;211(03):661-666

5 Ryu CW, Whang SM, Suh DC, et al. Percutaneous direct puncture glue embolization of high-flow craniofacial arteriovenous lesions: a new circular ring compression device with a beveled edge. AJNR Am J Neuroradiol 2007;28(03):528-530 
174 Percutaneous Endovascular Treatment of Scalp AVM Kannath, Rajan

6 Dabus G, Pizzolato R, Lin E, Kreusch A, Linfante I. Endovascular treatment for traumatic scalp arteriovenous fistulas: results with Onyx embolization. J Neurointerv Surg 2014;6(05):405-408

7 Arat A, Cil BE, Vargel I, et al. Embolization of high-flow craniofacial vascular malformations with Onyx. AJNR Am J Neuroradiol 2007; 28(07):1409-1414

8 Dalyai RTZ, Schirmer CM, Malek AM. Transvenous balloon-protected embolization of a scalp arteriovenous fistula using Onyx liquid embolic. Acta Neurochir (Wien) 2011;153(06):1285-1290

9 Thiex R, Wu I, Mulliken JB, Greene AK, Rahbar R, Orbach DB. Safety and clinical efficacy of Onyx for embolization of extracranial head and neck vascular anomalies. AJNR Am J Neuroradiol 2011; 32(06):1082-1086

10 Matsushige T, Kiya K, Satoh H, Mizoue T, Kagawa K, Araki H. Arteriovenous malformation of the scalp: case report and review of the literature. Surg Neurol 2004;62(03):253-259

11 Burrus TM, Miller GM, Flynn LP, Fulgham JR, Lanzino G. NeuroImages. Symptomatic left temporal arteriovenous traumatic fistula. Neurology 2009;73(07):570

12 Feng L, Mattson-Gates G, Sosa V, et al. P-018 curative transvenous embolisation of craniofacial arteriovenous malformation. J Neurolntervent Surg 2010;2(Suppl 1):A23 